\title{
A major genetic locus controlling natural Plasmodium falciparum infection is shared by East and West African Anopheles gambiae
} Michelle M Riehle*1, Kyriacos Markianos ${ }^{2}$, Louis Lambrechts ${ }^{3}$, Ai Xia ${ }^{4}$, Igor Sharakhov ${ }^{4}$, Jacob C Koella ${ }^{5}$ and Kenneth D Vernick ${ }^{1}$

\begin{abstract}
Address: ${ }^{1}$ Microbial and Plant Genomics Institute and Department of Microbiology, University of Minnesota, St. Paul, MN 55108, USA, ${ }^{2}$ Program in Genomics, Department of Medicine, Children's Hospital Boston, Harvard Medical School, Boston, MA 02115, USA, ${ }^{3}$ Department of Entomology, University of California, Davis, CA 95616, USA, ${ }^{4}$ Department of Entomology, Virginia Tech University, Blacksburg, VA 24061, USA
\end{abstract} and ${ }^{5}$ Division of Biology, Imperial College London, Ascot, Berkshire SL5 7PY, UK

Email: Michelle M Riehle* - mriehle@umn.edu; Kyriacos Markianos - kmarkianos@enders.tch.harvard.edu; Louis Lambrechts - llambrechts@ucdavis.edu; Ai Xia - xia@vt.edu; Igor Sharakhov - igor@vt.edu; Jacob C Koella - jkoella@imperial.ac.uk; Kenneth D Vernick - kvernick@umn.edu

* Corresponding author

Published: 6 July 2007

Malaria Journal 2007, 6:87 doi:10.1 I86/1475-2875-6-87

This article is available from: http://www.malariajournal.com/content/6/l/87

(C) 2007 Riehle et al; licensee BioMed Central Ltd.

This is an Open Access article distributed under the terms of the Creative Commons Attribution License (http://creativecommons.org/licenses/by/2.0), which permits unrestricted use, distribution, and reproduction in any medium, provided the original work is properly cited.
Received: 2 January 2007

Accepted: 6 July 2007

\begin{abstract}
Background: Genetic linkage mapping identified a region of chromosome 2L in the Anopheles gambiae genome that exerts major control over natural infection by Plasmodium falciparum. This $2 \mathrm{~L}$ Plasmodium-resistance interval was mapped in mosquitoes from a natural population in Mali, West Africa, and controls the numbers of $P$. falciparum oocysts that develop on the vector midgut. An important question is whether genetic variation with respect to Plasmodium-resistance exists across Africa, and if so whether the same or multiple geographically distinct resistance mechanisms are responsible for the trait.

Methods: To identify $P$ falciparum resistance loci in pedigrees generated and infected in Kenya, East Africa, 28 microsatellite loci were typed across the mosquito genome. Genetic linkage mapping was used to detect significant linkage between genotype and numbers of midgut oocysts surviving to $7-$ 8 days post-infection.

Results: A major malaria-control locus was identified on chromosome $2 \mathrm{~L}$ in East African mosquitoes, in the same apparent position originally identified from the West African population. Presence of this resistance locus explains $75 \%$ of parasite free mosquitoes. The Kenyan resistance locus is named EA_Pfin I (Éast $\underline{\text { Africa_ }}$ Plasmodium falciparum Infection Intensity).

Conclusion: Detection of a malaria-control locus at the same chromosomal location in both East and West African mosquitoes indicates that, to the level of genetic resolution of the analysis, the same mechanism of Plasmodium-resistance, or a mechanism controlled by the same genomic region, is found across Africa, and thus probably operates in A. gambiae throughout its entire range.
\end{abstract}

\section{Background}

The mosquito Anopheles gambiae is the major African vector of human malaria caused by Plasmodium falciparum.
More than 300 million cases of acute malaria occur globally each year resulting in over one million deaths with greater than $90 \%$ of these deaths occurring in children 
from Sub-Saharan Africa [1]. In malaria endemic countries where the poorest sections of society are most affected, inadequate public health initiatives and the selection of insecticide resistance hinder the efforts to control malaria transmission. Recent proposals to curb the malaria burden involve more technological approaches including the generation and introduction of transgenic mosquitoes [2]. However, all approaches have their own logistical, political and ethical limitations.

Here the genetics of natural vector resistance to $P$. falciparum is dissected, with the ultimate goal to utilize successful existing natural Plasmodium resistance mechanisms for control of human malaria transmission. Knowledge of the prevalence, strength and genomic location of loci that control mosquito resistance to malaria parasites in vector populations could aid future efforts to control parasite transmission. Genetically resistant and susceptible mosquitoes exist in nature, and phenotypic variation can segregate as a simple Mendelian trait [3]. A recent comprehensive study reported the prevalence, strength and genomic location of natural $P$. falciparum resistance loci in A. gambiae in Mali, West Africa [4]. The loci with the strongest phenotypic effect on parasite numbers clustered to form a Plasmodium-resistance island (PRI) on chromosome $2 \mathrm{~L}$. It would be valuable to know whether the genetic mechanism of the PRI identified in Mali is specific to that population, suggesting that there might be many geographically local $A$. gambiae resistance mechanisms across Africa, or conversely whether the mechanism identified by the PRI exists in A. gambiae throughout Africa. A major barrier to gene flow in A. gambiae occurs between West and East African populations [5], and thus comparing these populations should offer the most stringent test of the geographic generality of genetic loci controlling resistance mechanisms.

\section{Methods}

The source of the pedigrees was previously reported [6]. Briefly, isofemale pedigrees were initiated from a laboratory colony (established in Mbita, Kenya in 2001, approximately three years prior to use in this study) and raised to the F1 generation at which point they were challenged on gametocyte containing blood. The same pedigrees were previously used to investigate the role of genotype by genotype interactions in controlling the parasite load of vector mosquitoes [6]. Thus the parasite challenge was heterogeneous: each F1 pedigree was split into 3-5 groups that were each fed on independent natural gametocytemic blood samples. Unfed and partially fed females were excluded from further analysis. Seven to eight days after the infective blood meal, mosquito midguts were dissected and stained with $2 \%$ mercurochrome, and the numbers of oocysts were counted by light microscopy. Mosquito carcasses were saved for later DNA extraction.
DNA was isolated from mosquitoes using Qiagen DNeasy (Qiagen, CA, USA). Microsatellite genotyping was performed multiplexed as described previously [4]. Fragments were separated on an ABI3100 Genetic Analyzer and sized using GeneMapper Version 3.5 (both from Applied Biosystems, CA, USA). All automated genotype calls were visually verified. Mapping was carried out in those pedigrees deemed potentially informative using previously described guidelines; $\geq 20$ females with $\geq 30 \%$ infection prevalence [4]. Six pedigrees, named 57, 92, $141,142,146$ and 1102 met the typing criteria. Twentyeight microsatellite loci were screened in these informative pedigrees and as many as 15 additional loci were typed in some, but not all pedigrees (Figure 1). Many of these additional markers were added to increase the signal resolution around marker $\mathrm{H} 325$. All primer sequences are available elsewhere $[4,7]$ except 2L.24D left 5' TGCCAATCAATCAGTGTGCT $3^{\prime}$ and right 5' CACTAGCAACGGCACTACCC 3' and 2L.26D left 5' GAAGTGGAAGAACACGCTCA and right 5' CACACGATTGCAGATGAGTT 3'. Physical positions given are based on

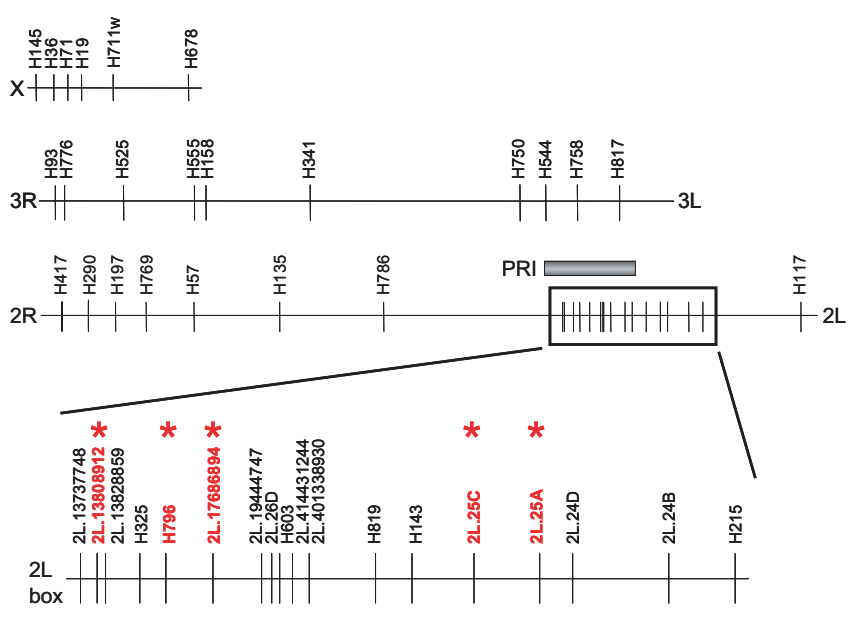

Figure I

Physical positions of microsatellite markers used in genetic mapping. The three chromosomes are indicated as horizontal lines (from top: X, 3, 2), and positions of marker loci are vertical lines labeled with the marker name. Note the high density of markers on chromosome $2 \mathrm{~L}$ (boxed section expanded in bottom horizontal line, labeled $2 \mathrm{~L}$ box), which were used to map at greater density once an initial linkage signal was detected. Dense markers were necessary so that the most informative markers (i.e., those with the greatest numbers of alleles segregating) could be used in the analysis. The five clustered marker loci giving significant linkage (linkage data shown in Table I) are depicted in bold red type and are starred. For comparison, the extent of the Plasmodiumresistance island mapped in West African pedigrees [4] is indicated by the filled bar above chromosome $2 \mathrm{~L}$ (labeled $\mathrm{PRI})$. Chromosome 2 is shown in the $2 \mathrm{La}$ inverted (2La/a) conformation. 
Ensembl A. gambiae genome sequence version 39, converted to the inverted $2 \mathrm{La} / \mathrm{a}$ form by use of inversion breakpoint coordinates from [8].

Linkage analysis methods are described in detail elsewhere [3]. Despite the fact that each pedigree was divided into groups that were fed on different gametocyte carriers, in the current study all individuals within a pedigree were first analysed together. In one pedigree yielding a linkage signal, the infections from different parasite isolates were also analysed individually. In brief, at every marker, the pedigree members were split into two samples, using an allele or genotype observed at that marker as the selection criterion. At each locus, the distribution of oocyst numbers for each allele/genotype was compared against the pooled oocyst distribution for all other alleles/genotypes. Permutations were used to establish empirical genomewide p-values. Wilcoxon-Mann-Whitney (WMW), a nonparametric statistical test, was used to determine statistical significance.

A novel and important aspect of this mapping methodolgy is that it examines each locus in isolation from all other loci and does not require knowledge of relative marker position. Thus, the result is robust to the presence of segregating chromosomal inversions, which are common in A. gambiae, and can alter the physical relationship between markers in different individuals within a pedigree. For example, another recent mapping study of $P$. falciparum resistance in other Kenyan pedigrees [9] used a mapping approach in which valid linkage results are dependent on correct knowledge of marker order, but marker order was not empirically determined. That study assumed a marker order [7] that predated release of the A. gambiae genome sequence. In fact, marker order in the genome sequence differs markedly from that in [7,9] (e.g., chromosome 2L ref [7,9] order: AG2H46-AG2H197AG2H786-AG2H135-AG2H603-AG2H787-AG2H325; genome order and current study: AG2H46-AG2H197AG2H135-AG2H786-AG2H325-AG2H787-AG2H603).

The statistical analysis was run using both the program OUTBRED_LINES as previously described [3] and a new implementation of the same algorithm in the statistical analysis environment R [10], called OUTBRED_LINES-R. The motivation was to avoid use of approximations employed in OUTBRED_LINES and instead use the builtin exact WMW tests. However, very similar results were obtained from OUTBRED_LINES and OUTBRED_LINES$\mathrm{R}$. Exact $\mathrm{p}$-values from the $\mathrm{R}$ implementation OUTBRED_LINES-R are presented throughout the manuscript.

\section{Results}

Six Kenyan pedigrees met the minimum informativeness criteria for genotyping ( $\geq 20$ mosquitoes surviving to dis- section, with infection prevalence $\geq 30 \%$ ), and these pedigrees were completely genotyped (Figure 2). One of the pedigrees ( $n=25$, pedigree \#57 in [6]) yielded a significant locus for $P$. falciparum resistance (nominal $\mathrm{p}=0.002$, genome-wide $\mathrm{p}=0.040$ ). The locus displays significant linkage to 5 nearby markers (genome wide $\mathrm{p}<0.05$; from left to right; 2L.H13808912, H796, 2L.17686896, 2L.25C, 2L.25A; Figure 1, Table 1). The Kenyan resistance locus is here named EA_Pfin1 (East Africa_ Plasmodium falciparum Infection Intensity), to distinguish it from the previously named Pfin1 locus, which it overlaps, identified in the chromosome 2L PRI of West African A. gambiae [3,4]. This mapping with greater marker density shows solid statistical support for the EA_Pfin1 resistance locus from multiple genetic markers (Figure 3 ). The detection of maximum linkage over a large physical region $(\sim 16 \mathrm{Mb})$ is due to the limited number of recombinants expected in such a small pedigree $(n=25)$. Despite small pedigree size, the EA_Pfin1 locus had high explanatory power for infection phenotype: the marker allele linked to resistance explained $75 \%$ of completely uninfected mosquitoes in the pedigree, and the susceptible allele explained $89 \%$ of oocyst-containing mosquitoes (Figure 4). The other five

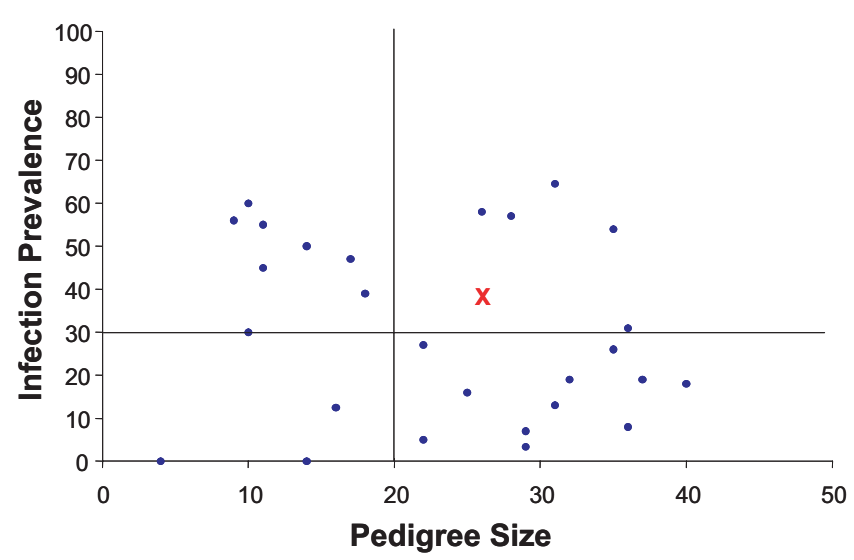

Figure 2

Pedigree criteria for genetic analysis. Pedigrees were chosen for genome-wide genotyping and linkage analysis based on unbiased criteria of infection prevalence (the percent of mosquitoes with at least one midgut oocyst) and pedigree size. These criteria, previously applied in the West African pedigree study [4], prioritize the full analysis of the potentially most informative pedigrees. Pedigrees with $\geq 20$ mosquitoes were analysed (actual range 25-36) and $\geq 30 \%$ infection prevalence (actual range, 31-64\%). Only 6 of 28 pedigrees met this criterion, shown in the upper right quadrant. Pedigrees falling outside these boundaries are unlikely to yield a detectable genetic signal, even if segregating strong alleles for resistance/susceptibility. The horizontal line inside the graph indicates $30 \%$ prevalence and the vertical line indicates a pedigree size of 20 . The red $x$ indicates the pedigree that gave a significant linkage signal. 
informative pedigrees gave no significant linkage signal (discussed below).

Careful examination of the EA_Pfin 1 pedigree indicates that one of the three parasite isolates used failed to infect any mosquitoes in the pedigree, and infected only five mosquitoes of 76 total assayed across all pedigrees [6]. Due to the lack of infectivity of the parasites in this blood sample for the EA_Pfin1 pedigree, the data from mosquitoes fed on this isolate were removed and the pedigree was reanalysed. Without these six mosquitoes, the remaining 19 mosquitoes still give significant linkage signal at the same microsatellite loci despite the small sample size (Table 2). This result indicates that the linkage signal did not depend solely or disproportionately upon defeat by the mosquitoes of one of the three parasite isolates fed to them. Indeed, even with loss of $24 \%$ of the pedigree sample size by removal of the six mosquitoes, the linkage signal was still comparable to that from the entire pedigree. The surviving linkage signal in the 19 mosquitoes was due to mosquitoes in the pedigree whose parasite challenge was divided between two different gametocyte carriers. Analysis of the remaining two groups fed on either gametocyte carrier alone did not yield a linkage signal (not shown).

Thus, the detected linkage signal in the entire pedigree or any subset did not result from a strong protective effect by mosquitoes against any single parasite isolate. Instead, detection of linkage required the larger sample size obtained by combining groups of mosquitoes that fed on at least two different parasite isolates, and consequently the signal must have originated in the pedigree's consistent genetic response to at least two different parasite isolates. Taken together, these results indicate that Plasmodium resistance controlled by the A. gambiae EA_Pfin1 locus can be independent of and transcend parasite genotype.

\section{Discussion}

A P. falciparum resistance locus, EA_Pfin1, is reported in East African A. gambiae that resides within the chromo- somal Plasmodium-resistance Island (PRI) detected in West African A. gambiae. The phenotype is also the same: parasite elimination prior to oocyst development. The vast majority ( $>75 \%$ ) of mosquitoes in the EA_Pfin 1 pedigree carrying the resistance allele completely blocked parasite development, and thus were parasite-free. This is comparable to the phenotypic effect size of resistance loci in the 2L PRI in West Africa [4]. Due to their chromosomal co-localization and similarity of phenotype, EA_Pfin 1 and the 2L PRI in West Africa likely control the same underlying Plasmodium resistance mechanism.

\section{Geographic generality of resistance}

The geographic generality of resistance mechanisms indicates that the PRI-controlled mechanism of resistance is not a nuance of the pedigrees derived from the West Africa population initially studied. Another recent study in Kenya [9] detected an effect on $P$. falciparum infection outcome, but physical positions of the markers and therefore the mapped loci in that study were not determined and thus it is not possible to resolve whether the effect was controlled by the same genomic region as reported here. Most parsimoniously, a common resistance mechanism across the African continent suggests a relatively ancient origin for natural $P$. falciparum resistance rather than the evolution of distinct resistance mechanisms multiple times. The ancestral PRI-controlled mechanism might have been selected in an A. gambiae ancestor by exposure to a non-Plasmodium pathogen, or might have newly evolved in A. gambiae soon after the advent of the P. falciparum host-pathogen system.

\section{Factors on chromosome 2 L control malaria parasite infection}

Genetic factors on chromosome 2L have previously been shown to affect malaria infection both in nature [11] and in a laboratory system $[12,13]$. In addition, the form of the polymorphic 2La inversion is also correlated with adaptation to climate [14], biting and resting behavior [15], and morphology [16]. The previous mapping pedigrees from West Africa were all fixed inverted homozygotes $(2 \mathrm{La} / \mathrm{a})$ for the $2 \mathrm{La}$ inversion [4]. A molecular

Table I: Mapping of the EA_Pfin I locus.

\begin{tabular}{|c|c|c|c|c|c|c|}
\hline Marker & Position on $2 \mathrm{~L}$ & \# of Alleles & $\begin{array}{l}\text { Allele Linked to } \\
\text { High Oocyst \# }\end{array}$ & $\begin{array}{c}\text { Allele Linked to Low } \\
\text { Oocyst \# }\end{array}$ & $\begin{array}{l}\text { Average \# of Oocysts } \\
\text { with Susceptible Allele }\end{array}$ & $\begin{array}{l}\text { Average \# of Oocysts } \\
\text { with Resistant Allele }\end{array}$ \\
\hline 2L. 13808912 & I3.8 MB & 3 & 87 & n.d. & 2.92 & n.d. \\
\hline $\mathrm{H} 796$ & 16.7 MB & 4 & 84 & 82 & 2.92 & 0.08 \\
\hline 2L. I 7686894 & $18.3 \mathrm{MB}$ & 4 & 189 & 180 & 2.92 & 0.08 \\
\hline $2 \mathrm{~L} .25 \mathrm{C}$ & $26.6 \mathrm{MB}$ & 4 & 72 & 132 & 2.50 & 0.09 \\
\hline 2L.25A & $29 \mathrm{MB}$ & 3 & n.d. & 160 & n.d. & 0.08 \\
\hline
\end{tabular}

Linkage data for the five microsatellite markers significantly linked with $P$. falciparum infection outcome (genome-wide $p$ value $<0.05$ ). Physical positions are based on the inverted $2 \mathrm{La} / \mathrm{a}$ chromosome form. Other markers in this region did not yield significant linkage signal in this pedigree due to a low level of marker informativeness. Because 4 distinct chromosomes were segregating, as indicated by most markers, the two markers with 3 alleles were not fully informative because I allele marked 2 chromosomes, thus obscuring the linkage signal from the non-informative cases (labeled n.d., not detected). The nominal and genome-wide $p$ values for all markers listed in the table are 0.002 and 0.040, respectively 


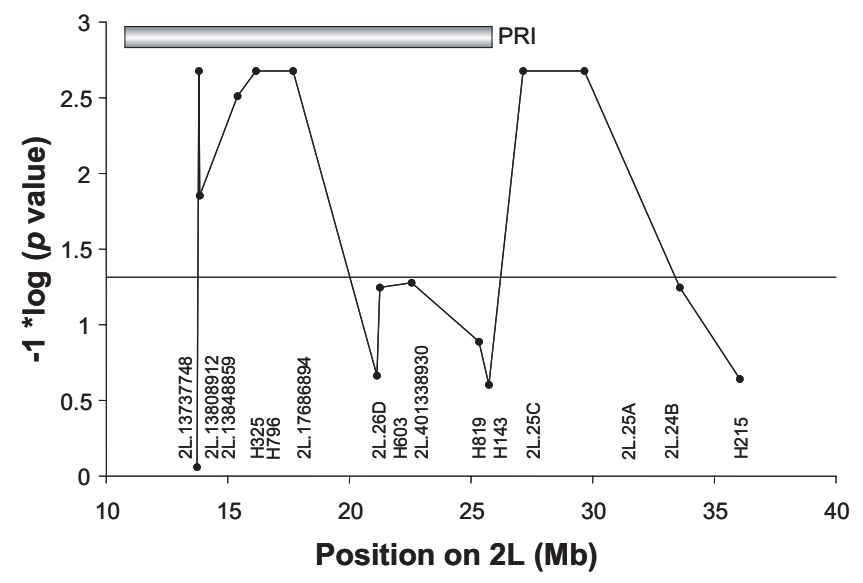

Figure 3

Mapping of the chromosomal region containing EA_Pfin I. Nominal $p$ values are plotted as a function of physical position. For reference, a value of I.3 on the transformed $y$-axis corresponds to a $p$ value of 0.05 , shown as a horizontal line. The order of markers uses the inverted ( $2 \mathrm{La} /$ a) conformation of the $2 \mathrm{~L}$ chromosome. Only the most informative markers ( $\geq 2$ alleles and $\geq 3$ genotypes) are shown. Only markers H796, HI7686896, and H25C are fully informative, that is, segregate 4 alleles so that a unique microsatellite allele marks each parental chromosome that entered the pedigree. The dip in the center of the plot is likely due to a lack of marker informativeness.

diagnostic assay designed to determine the orientation of the breakpoints of the 2La inversion [8] indicated that the EA_Pfin1 pedigree in the current study is similarly fixed for the inverted chromosome (data not shown).

Therefore, the data show that at least one strong resistance locus segregates within the inverted form of 2La across Africa. Without homozygous 2La standard form $(2 \mathrm{La}+/+)$ pedigrees or pedigrees with the $2 \mathrm{La}$ inversion segregating (which would include heterozygotes and both homozygotes) the data cannot address whether there is linkage between inversion state and parasite infection loads. Given the lack of any pedigrees in the West African studies fixed for or segregating the $2 \mathrm{La}+/+$ standard form arrangement, and the colony origin and small size of the current East African pedigrees, it is also impossible to know whether this PRI-related mechanism of $P$. falciparum resistance is unique to the inverted form or common across forms. In a confusing turn of nomenclature, the inverted arrangement of 2La is actually ancestral, and the standard form is derived [8]. Thus, it is formally possible that the monophyletic inversion event leading to the "standard" chromosome $(2 \mathrm{La}+/+)$ could have captured a susceptible allele from the PRI. Another mapping study done in East Africa cannot be compared because it did not report the inversion state of 2La [9].

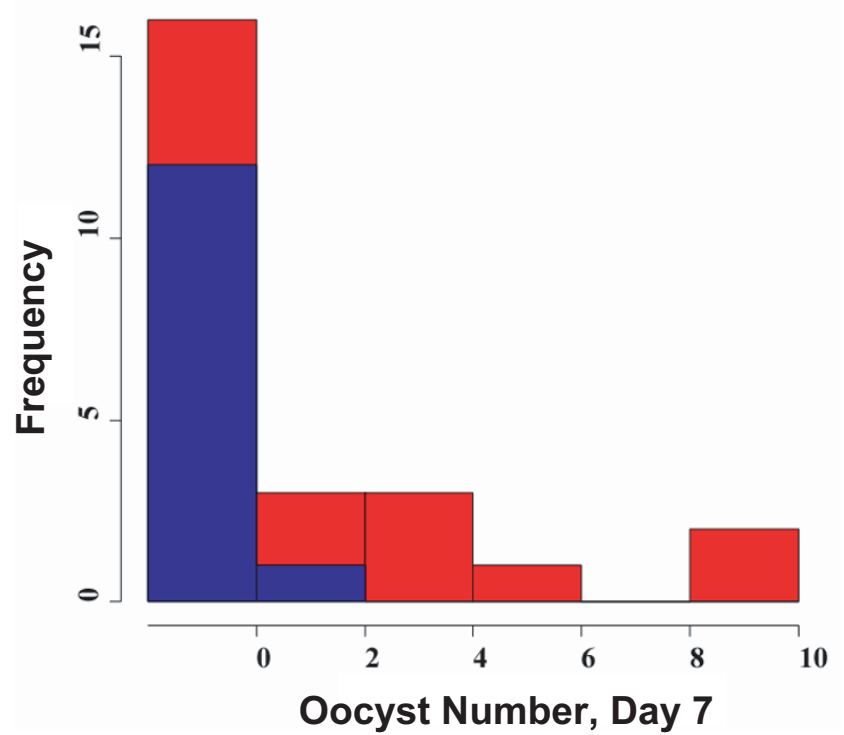

Figure 4

Oocyst distribution and allele frequency at microsatellite marker H796. Four alleles are segregating at H796, but only two are informative for infection outcome. Allele 82 (blue) is linked to low oocyst number (average oocyst number 0.08) and allele 84 (red) is linked to high oocyst number (average oocyst number 2.9). Similar histograms can be drawn for the other loci yielding significant linkage with infection outcome.

\section{Resistance alleles maintained in colonies}

Prior linkage mapping $[3,4]$ of $P$. falciparum resistance loci used F1 or F2 pedigrees that were only a generation or two removed from the wild, while the current study uses pedigrees generated from a colony established several years earlier. It is important to emphasize the importance of detecting resistance loci in a natural population versus observation in a colony, where selection and genetic drift can have a strong influence on the prevalence of susceptibility/resistance alleles. Repeated observations in pedigrees derived from the natural mosquito population [4] is a reliable proxy for prevalence of resistance/susceptibility

Table 2: Parasite strain transcendence. Comparison of nominal and genome wide $\mathrm{p}$ values for linkage at $\mathrm{H} 796$ before and after removing mosquitoes fed on a parasite isolate that did not infect any mosquitoes in the pedigree.

\begin{tabular}{ccc}
\hline H796 & \multicolumn{2}{c}{$\boldsymbol{P}$ value } \\
\hline & All parasites & Only infective parasites \\
\hline Nominal & 0.002 & 0.003 \\
Genome-wide & 0.040 & 0.035
\end{tabular}

To obtain $p$ values for only infective parasites, those mosquitoes that fed on the parasite isolate that failed to infect any mosquitoes were removed prior to analysis ( $n=6$, leaving 19 mosquitoes for analysis) 
in the field. In contrast, breeding in a colony can amplify or diminish the prevalence of the relevant alleles. Thus, it is only in light of the previous West African results [3,4], as well as a consistent observation from an independent effort [9], that it can be concluded that the chromosome 2L PRI is indeed a common resistance mechanism in the Kenyan field population.

Detection of $P$. falciparum resistance alleles in a colonyderived pedigree demonstrates that despite the large loss of genetic diversity that accompanies colonization [17], and the absence of any putative parasite-imposed selective pressure for many generations, alleles for resistance and susceptibility to parasite infection can still segregate within a colony. The ability of resistant and susceptible alleles to persist in a colony setting is advantageous for future mechanistic studies of natural genetic resistance factors.

\section{Parasite strain transcendence}

The current mapping study allowed testing for effects across parasite isolates within a mosquito pedigree. Detection of linkage in a pedigree in which different mosquitoes were fed on multiple distinct parasite isolates is consistent with the fact that the allele for resistance in the EA_Pfin1 mapping pedigree controls an effect that transcends parasite strain. This is based on the assumption of non-relatedness between parasite isolates, an assumption which is consistent with the previous analysis of the same infected pedigrees [6]. Parasite strain transcendence or independence is evident from the genetic mapping in West Africa [4], in which multiple independent isofemale pedigrees over multiple years, each fed on blood from a different gametocyte carrier, nevertheless yielded loci in the same 2La genomic region.

\section{Conclusion}

Here it is established that the genomic region of A. gambiae chromosome $2 \mathrm{~L}$, containing strong Plasmodiumresistance loci originally identified in pedigrees from a West Africa population of vector mosquitoes, is not a geographically local phenomenon. At the level of resolution of the genetic mapping, the same genetic factor also exists in East African A. gambiae. The intriguing prospect of shared $P$. falciparum resistance loci across Africa makes it a promising approach to consider taking advantage of widespread natural Plasmodium resistance mechanisms for the control of malaria transmission $[18,19]$.

\section{Authors' contributions}

This work was a collaborative effort involving all authors. LL initiated and infected the Kenyan pedigrees and JK provided DNA samples from them. MMR performed genotyping assays and statistical analysis. KM performed statistical analyses. IS and AX typed the pedigree yielding a linkage signal for the 2La inversion. MMR, KM, and KV discussed analysis of results and interpreted their meaning. Both MMR and KM wrote initial drafts of the manuscript. The final draft of the ms was read and approved by all authors.

\section{Acknowledgements}

This work was supported by grants from NIAID (KDV), NHGRI (KM), and was aided by resources of the University of Minnesota Supercomputing Institute Computational Genetics Laboratory. The assistance of Dr. Louis Gouagna and the staff of the Malaria Department at ICIPE-Mbita is acknowledged for their assistance during the generation and experimental infection of the pedigrees, and for helpful comments on the manuscript. Informed consent was obtained for studies on humans after the nature and possible consequences of the studies were explained.

\section{References}

I. World Health Organization [http://www.who.int/tdr/diseases/ malaria]

2. Christophides GK: Transgenic mosquitoes and malaria transmission. Cell Microbiol 2005, 7(3):325-333.

3. Niare O, Markianos K, Volz J, Oduol F, Toure A, Bagayoko M, Sangare D, Traore SF, Wang R, Blass C, Dolo G, Bouare M, Kafatos FC, Kruglyak L, Toure YT, Vernick KD: Genetic loci affecting resistance to human malaria parasites in a West African mosquito vector population. Science 2002, 298(559I):2/3-2I6.

4. Riehle MM, Markianos K, Niare O, Xu J, Li J, Toure AM, Podiougou B, Oduol F, Diawara S, Diallo M, Coulibaly B, Ouatara A, Kruglyak L, Traore SF, Vernick KD: Natural malaria infection in Anopheles gambiae is regulated by a single genomic control region. Science 2006, 3 I 2(5773):577-579.

5. Lehmann T, Licht M, Elissa N, Maega BT, Chimumbwa JM, Watsenga FT, Wondji CS, Simard F, Hawley WA: Population Structure of Anopheles gambiae in Africa. J Hered 2003, 94(2): I33-I 47.

6. Lambrechts L, Halbert J, Durand P, Gouagna LC, Koella JC: Host genotype by parasite genotype interactions underlying the resistance of anopheline mosquitoes to Plasmodium falciparum. Malar J 2005, 4:3.

7. Zheng L, Benedict MQ, Cornel AJ, Collins FH, Kafatos FC: An integrated genetic map of the African human malaria vector mosquito, Anopheles gambiae. Genetics 1996, I43:941-952.

8. Sharakhov IV, White BJ, Sharakhova MV, Kayondo J, Lobo NF, Santolamazza F, Della Torre A, Simard F, Collins FH, Besansky NJ: Breakpoint structure reveals the unique origin of an interspecific chromosomal inversion (2La) in the Anopheles gambiae complex. Proc Natl Acad Sci U S A 2006, I03(16):6258-6262.

9. Menge DM, Zhong D, Guda T, Gouagna L, Githure J, Beier J, Yan G: Quantitative trait loci controlling refractoriness to Plasmodium falciparum in natural Anopheles gambiae mosquitoes from a malaria-endemic region in western Kenya. Genetics 2006, I73(I):235-24I.

10. R Development Core Team: R: A language and environment for statistical computing. R Foundation for Statistical Computing [http:l /www.R-project.org].

II. Petrarca V, Beier JC: Intraspecific chromosomal polymorphism in the Anopheles gambiae complex as a factor affecting malaria transmission in the Kisumu area of Kenya. Am J Trop Med Hyg 1992, 46(2):229-237.

12. Vernick KD, Collins FH: Association of a Plasmodium-refractory phenotype with an esterase locus in Anopheles gambiae. Am J Trop Med Hyg 1989, 40(6):593-597.

13. Crews-Oyen AE, Kumar V, Collins FH: Association of two esterase genes, a chromosomal inversion, and susceptibility to Plasmodium cynomolgi in the African malaria vector Anopheles gambiae. Am J Trop Med Hyg 1993, 49(3):34I-347.

14. Toure YT, Petrarca V, Traore SF, Coulibaly A, Maiga HM, Sankare O, Sow M, Di Deco MA, Coluzzi M: The distribution and inversion polymorphism of chromosomally recognized taxa of the Anopheles gambiae complex in Mali, West Africa. Parassitologia 1998, 40(4):477-5II. 
15. Rishikesh N, Di Deco MA, Petrarca V, Coluzzi M: Seasonal variations in indoor resting Anopheles gambiae and Anopheles arabiensis in Kaduna, Nigeria. Acta Trop 1985, 42(2): 165- 170.

16. Petrarca V, Sabatinelli G, Di Deco MA, Papakay M: The Anopheles gambiae complex in the Federal Islamic Republic of Comoros (Indian Ocean): some cytogenetic and biometric data. Parassitologia 1990, 32(3):37I-380.

17. Norris DE, Shurtleff AC, Toure YT, Lanzaro GC: Microsatellite DNA polymorphism and heterozygosity among field and laboratory populations of Anopheles gambiae ss (Diptera: Culicidae). J Med Entomol 200I, 38(2):336-340.

18. Hahn MW, Nuzhdin SV: The fixation of malaria refractoriness in mosquitoes. Curr Biol 2004, I4(7):R264-265.

19. Hurd H, Taylor PJ, Adams D, Underhill A, Eggleston P: Evaluating the costs of mosquito resistance to malaria parasites. Evolution Int J Org Evolution 2005, 59( I 2):2560-2572.

Publish with Bio Med Central and every scientist can read your work free of charge

"BioMed Central will be the most significant development for disseminating the results of biomedical research in our lifetime."

Sir Paul Nurse, Cancer Research UK

Your research papers will be:

- available free of charge to the entire biomedical community

- peer reviewed and published immediately upon acceptance

- cited in PubMed and archived on PubMed Central

- yours - you keep the copyright

Submit your manuscript here:

http://www.biomedcentral.com/info/publishing_adv.asp 